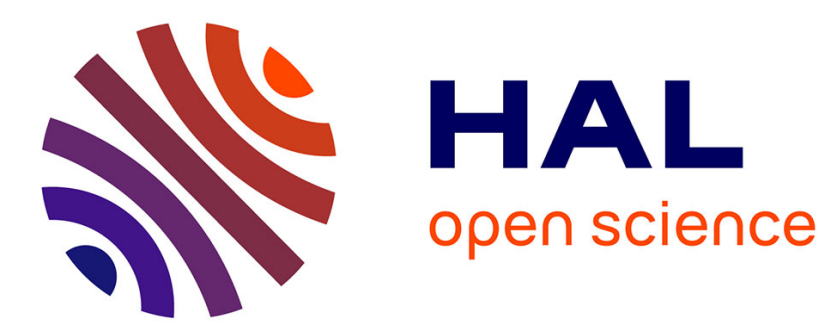

\title{
Réalisation d'un spectro-goniométre pour la diffraction des électrons de faible énergie
}

Ch. Burggraf, S. Goldsztaub, A. Mosser

\section{To cite this version:}

Ch. Burggraf, S. Goldsztaub, A. Mosser. Réalisation d'un spectro-goniométre pour la diffraction des électrons de faible énergie. Revue de Physique Appliquée, 1971, 6 (3), pp.319-323. 10.1051/rphysap:0197100603031900 . jpa-00243549

HAL Id: jpa-00243549

https://hal.science/jpa-00243549

Submitted on 1 Jan 1971

HAL is a multi-disciplinary open access archive for the deposit and dissemination of scientific research documents, whether they are published or not. The documents may come from teaching and research institutions in France or abroad, or from public or private research centers.
L'archive ouverte pluridisciplinaire HAL, est destinée au dépôt et à la diffusion de documents scientifiques de niveau recherche, publiés ou non, émanant des établissements d'enseignement et de recherche français ou étrangers, des laboratoires publics ou privés. 


\title{
RÉALISATION D'UN SPECTRO-GONIOMÈTRE POUR LA DIFFRACTION DES ÉLECTRONS DE FAIBLE ÉNERGIE
}

\author{
Ch. BURGgRAF, S. GOLDSZTAUB et A. MOSSER
}

Laboratoire de Minéralogie de Strasbourg

Equipe de Recherche Associée au C. N. R. S.

(Reçu le 2 mars 1971)

\begin{abstract}
Résumé. - Description d'un spectro-goniomètre à cage de Faraday. Celle-ci peut êtıe déplacée sur une surface sphérique, permettant de mesurer en fonction de la position angulaire l'intensité d'un flux d'électrons de faible énergie diffractés élastiquement et inélastiquement.

Application de cet appareil à l'étude de la répartition énergétique des électrons dans une réflexion de Bragg.

Abstract. - We describe a device with a Faraday cage which can be moved on a spherical surface. It allows to measure, versus the angular position, the intensity of a flux of low energy electrons which have been elastically or inelastically scattered.

This apparatus has been used to study the energy distribution of electrons in a Bragg reflexion
\end{abstract}

Introduction. - Lors de l'étude des surfaces cristallines par diffraction des électrons de faible énergie, la mesure des intensités et énergies diffusées dans les différentes directions prend une importance de plus en plus grande.

Sur les appareils de diffraction classiques, cette mesure se fait généralement à l'aide d'un photomètre qui mesure la brillance d'un écran fluorescent. Cette méthode présente un certain nombre d'inconvénients dus à l'inhomogénéité du dépôt sur l'écran aux défauts de sphéricité des grilles et à la présence de lumière parasite.

En vue d'éviter ces inconvénients, nous avons conçu et réalisé un appareil équipé d'une cage de Faraday mobile autour de deux axes perpendiculaires qui permet la mesure directe de l'intensité et de l'énergie diffusée par le cristal dans toutes les directions. Il est possible après effacement de l'échantillon de mesurer l'intensité et la répartition de l'énergie du faisceau direct, ce qui permet d'étalonner en valeur absolue les intensités diffractées. Ce système permet également le repérage angulaire des faisceaux diffusés au moyen de deux cercles divisés.

Ce dispositif est monté avec une optique électronique classique à trois grilles sur une bride standard de diamètre 8 pouces et peut par conséquent être adapté sans difficulté sur un diffracteur.

Des appareils de diffraction d'électrons lents à cylindre de Faraday mobile de types très divers ont été déjà réalisés [1], [2], [3]. Ils ne permettent pas d'explorer une sphère entière et par conséquent on ne peut comparer l'intensité d'une réflexion à celle du faisceau incident.
I. Description de l'appareil. - 1. OPTIQUE. L'appareil est équipé d'une optique électronique classique montée en porte-à-faux pour permettre la rotation de $190^{\circ}$ du bras supportant la cage de Faraday (Fig. 1a,b).

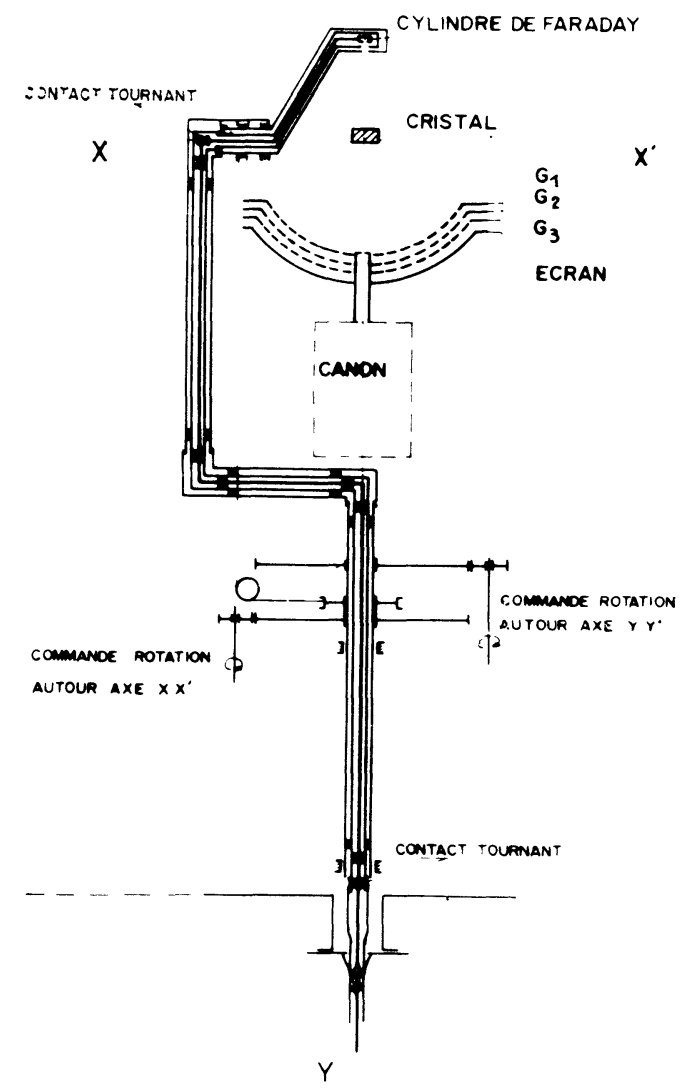

FIg. 1a. - Schéma de principe de l'appareil à cage de Faraday. 


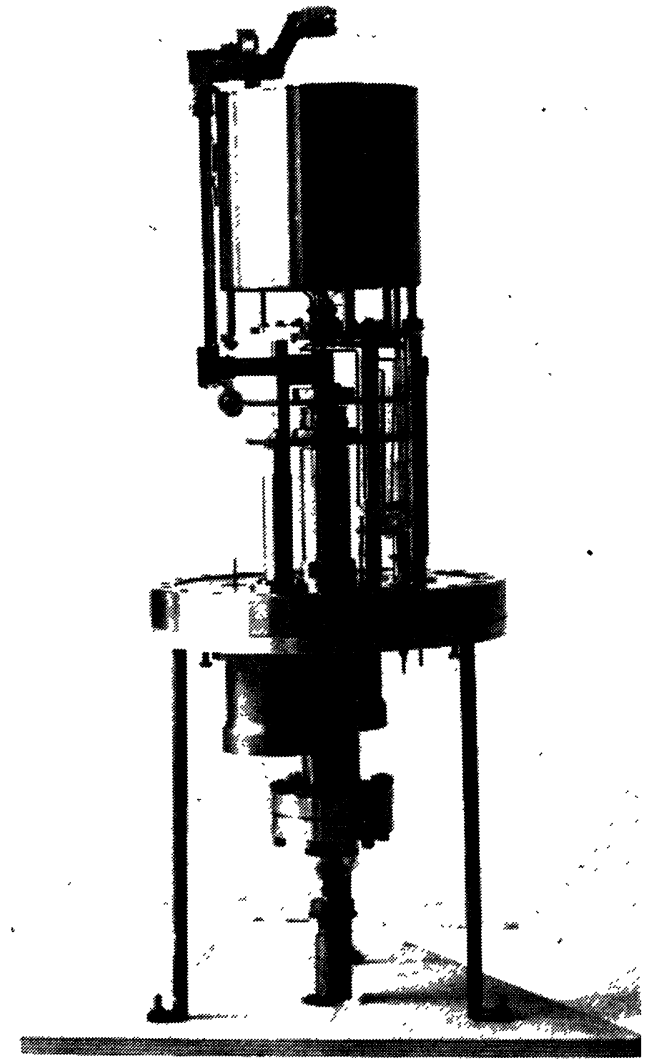

Fig. 1b. - Vue de l'appareil monté sur flasque standard.

L'écran fluorescent $E$ et les trois grilles $G_{1}, G_{2}$ et $G_{3}$ qui le précèdent ont la forme de calottes sphériques. La présence de la troisième grille permet de faire des mesures de spectroscopie "Auger». A cette fin, les amenées de courant de l'écran $E$ et de la grille $G_{2}$ ont été soigneusement blindées. Le canon à électrons spécialement étudié pour des électrons de faible énergie a été décrit dans un précédent article [4]. Il permet de travailler avec des électrons d'énergie comprise entre 20 et $3000 \mathrm{eV}$.

2. SYSTÈME DE MESURE. - a) But et principe. - Le spectro-goniomètre proprement dit est constitué par une cage de Faraday mobile autour de deux axes perpendiculaires $\mathrm{XX}^{\prime}$ et $\mathrm{YY}^{\prime}$ ce qui permet de le déplacer sur une sphère entière de $30 \mathrm{~mm}$ de rayon, centrée sur le cristal. On peut ainsi mesurer l'intensité et discriminer l'énergie des électrons suivant les différentes directions sans changer la position du faisceau incident par rapport au cristal.

La rotation de la cage de Faraday autour de l'axe $Y^{\prime}{ }^{\prime}$ qui est confondu avec l'axe du canon de l'appareil est limitée à $190^{\circ}$ environ par suite du montage en porte-àfaux de l'optique. Cette rotation est commandée de l'extérieur par un système à engrenages.

L'axe de rotation $\mathrm{XX}^{\prime}$ coupe l'axe $\mathrm{YY}^{\prime}$ sur le cristal au point d'impact du faisceau d'électrons primaire. Le mouvement de rotation autour de l'axe $\mathrm{XX}^{\prime}$ dont l'amplitude est supérieure à $360^{\circ}$ est commandé par un fil d'acier s'enroulant sur un système de poulies qui est commandé lui-même depuis l'extérieur de l'appareil par un système rotatif à engrenages identique au précédent. Un ressort maintient la tension du fil constante et supprime ainsi le jeu.

b) Commandes extérieures. - Pour rendre les deux mouvements autour de $\mathrm{XX}^{\prime}$ et $\mathrm{YY}^{\prime}$ indépendants, la commande du déplacement de la cage de Faraday est munie d'un embrayage (Fig. 2) ; lorsqu'il est débrayé la cage tourne autour de l'axe $\mathrm{XX}^{\prime}$, lorsqu'il est embrayé la cage se déplace autour de $Y^{\prime}$ '. Pour réaliser cette rotation, il faut que la poulie qui commande la rotation $\mathrm{XX}^{\prime}$ tourne du même angle et dans le même sens que $Y Y^{\prime}$. Ceci est obtenu par un choix convenable des couples d'engrenage.

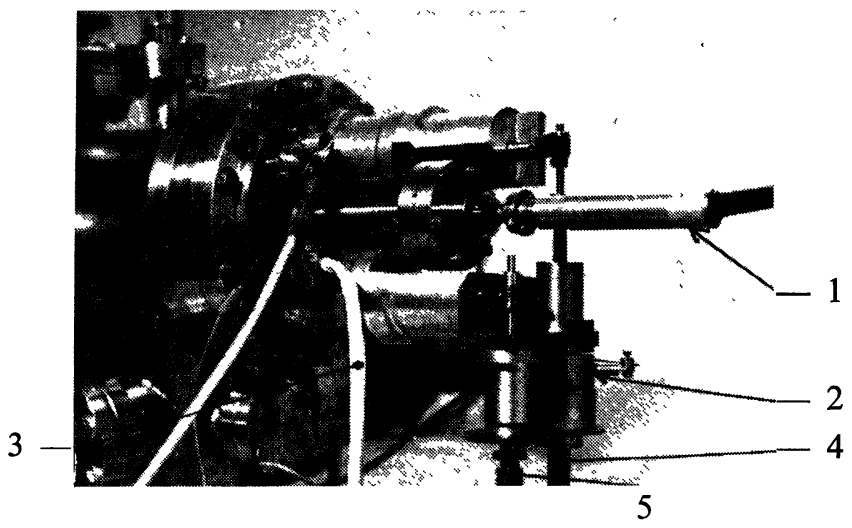

Fig. 2. - Système de commande de la cage de Faraday. 1. Conducteur coaxial de mesure ; 2 . Embrayage ; 3 . Alimentation du canon ; 4-5. Commandes de la cage de Faraday.

c) Cage de Faraday. - La cage de Faraday (Fig. 3) est constituée par deux cylindres coaxiaux enfermés dans une boîte parallélépipédique supportant le diaphragme d'entrée (diamètre $0,3 \mathrm{~mm}$ ).

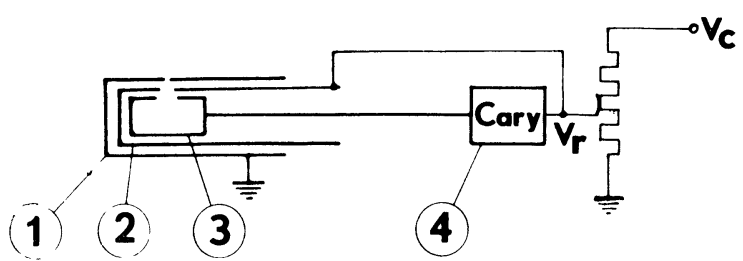

Fig. 3. - Schéma de la cage de Faraday utilisée avec potentiel retardateur. 1. Blindage extérieur; 2. Blindage intérieur (au même potentiel que 3) ; 3. Cage de Faraday ; 4. Electromètre à condensateur vibrant $«$ Cary $» V_{\mathrm{c}}=$ potentiel cathode ; $V_{\mathrm{r}}=$ potentiel retardateur.

Le cylindre interne formant la cage de Faraday proprement dite est percé d'un trou de diamètre $1,2 \mathrm{~mm}$ alors que le trou du cylindre de blindage n'est que de $1,0 \mathrm{~mm}$. Les trois trous sont alignés sur un même axe.

Une étude du dispositif de filtrage des électrons a montré que les dimensions indiquées ci-dessus n'étaient pas très critiques. De petites variations des côtes du système analyseur n'entra înent pas de variation perceptible dans la mesure de la dispersion du faisceau 
primaire du canon. Par contre, la dispersion énergétique dans le faisceau primaire est très sensible aux conditions de fonctionnement du canon et est souvent plus grande que la dispersion thermique de la cathode.

Le diffracteur d'électrons lents équipé de ce dispositif de mesure est représenté sur la figure 4.

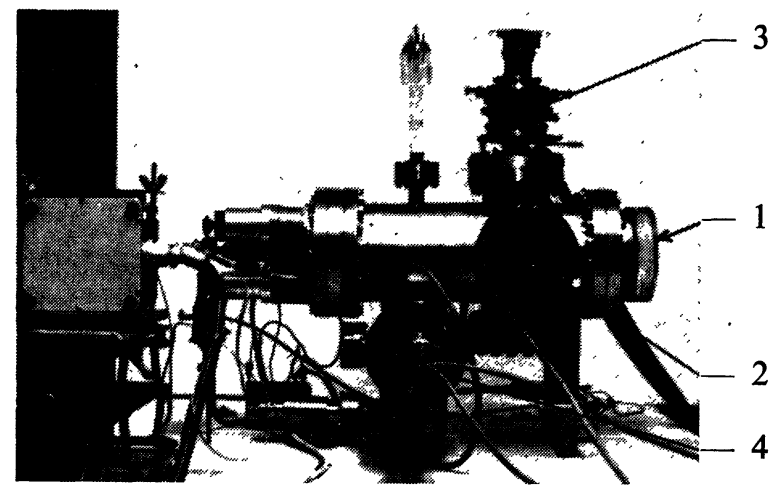

Fig. 4. - Vue d'ensemble du diffracteur d'électrons lents. 1. Fenêtre d'observation de l'écran ; 2. Fenêtre latérale servant au réglage du cristal ; 3 . Porte-cristal ; 4 . Evaporateur de titane.

d) Contacts tournants. - Etant donné la faible intensité à mesurer, il faut éviter tout courant parasite. Pour cela il importe qu'aucun isolant ne soit soumis à des contraintes variables, et que la capacité du circuit de mesure ne varie pas lors du déplacement de la cage de Faraday. Pour satisfaire cette condition les contacts tournants ont été réalisés de la façon représentée par la figure 5 . Le contact sphère-plan réduit la surface de frottement au minimum ; on a constaté que lors du déplacement continu de la cage de Faraday à l'aide du moteur, les perturbations introduites par l'appareil sont inférieures à $10^{-14} \AA$.

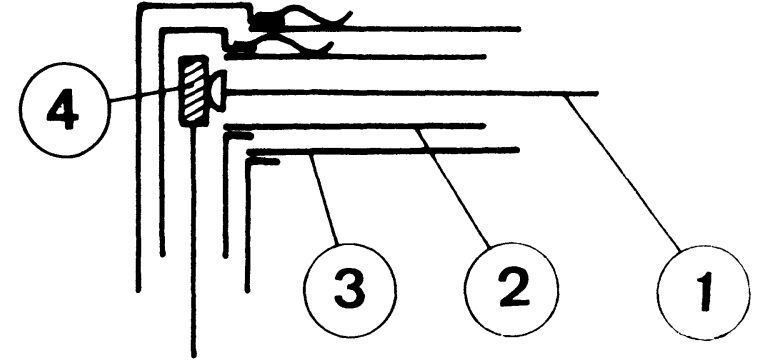

Fig. 5. - Schéma d'un contact tournant. 1. Conducteur de mesure ; 2. Blindage interne; 3 . Blindage extérieur ; 4. Contact plan-sphère.

e) Isolement. - L'isolement entre le conducteur central et le blindage interne est réalisé par des passages de courant en verre. Nous avons mesuré une résistance d'isolement supérieure à $10^{12} \Omega$, l'appareil étant assemblé.

L'isolement entre le blindage interne et le blindage externe est réalisé à l'aide de petites tiges d'alumine. Il supporte des différences de potentiel de $3000 \mathrm{~V}$.

II. Possibilités et limites de l'appareil. - 1. Mesure DES INTENSITÉs. - Le courant électronique recueilli par le cylindre de Faraday est mesuré par un électromètre à condensateur vibrant de type "Cary » qui permet de mesurer facilement les intensités comprises entre $10^{-6}$ et $10^{-14} \AA$. Il est possible d'enregistrer de façon continue l'intensité électronique en fonction du déplacement angulaire de la cage de Faraday grâce à un moteur synchrone.

La figure 6 montre l'enregistrement obtenu en déplaçant la cage de Faraday sur un petit cercle passant par les trois maxima de Bragg donnés par un cristal d'argent taillé suivant (111) (Fig. 7).

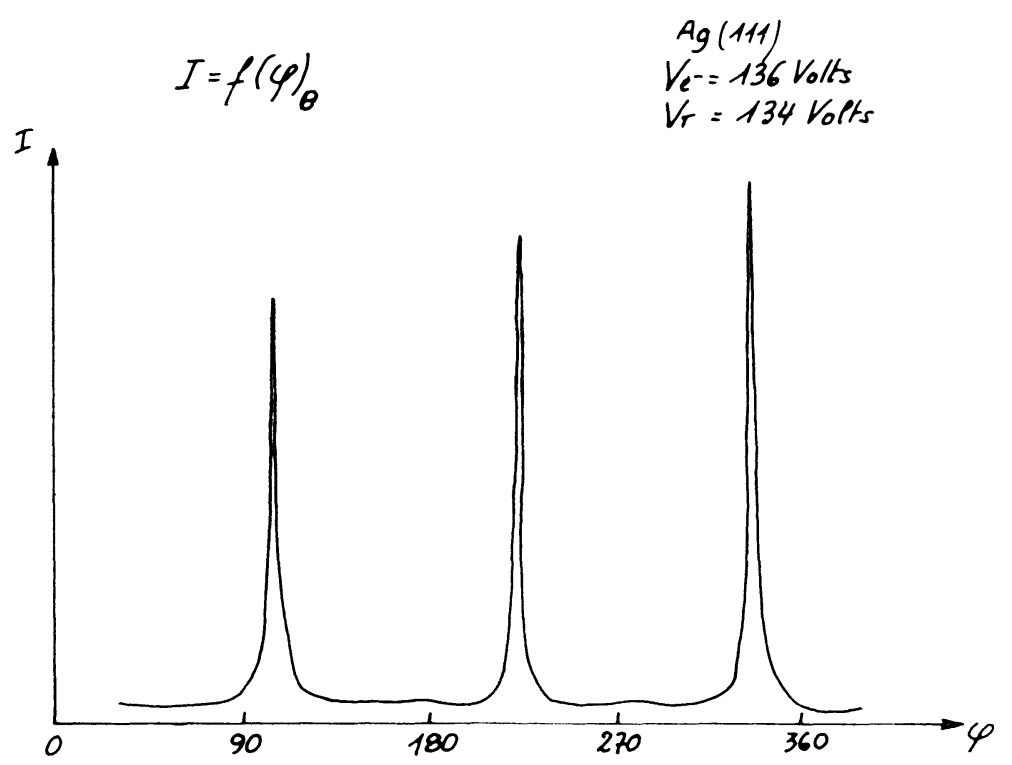

FIG. 6. - Distribution de l'intensité des électrons quasi-élastiques le long d'un petit cercle passant par les trois maxima de Bragg. 


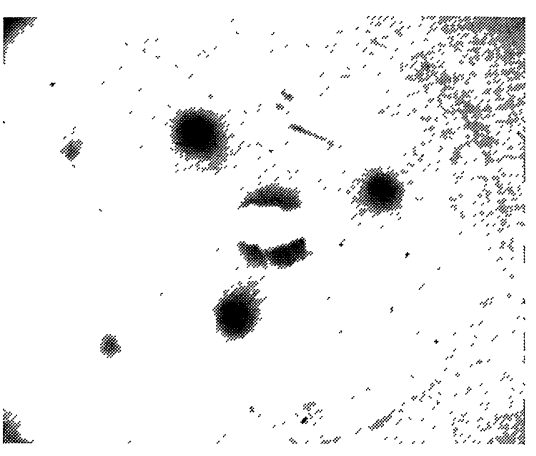

FIG. 7. - Diagramme de diffraction obtenu avec des électrons d'une énergie de $136 \mathrm{eV}$ sur une face (111) de l'argent.

La figure 8 montre la répartition de l'intensité pour différents potentiels retardateurs le long d'un cercle méridien passant par une réflexion de Bragg.

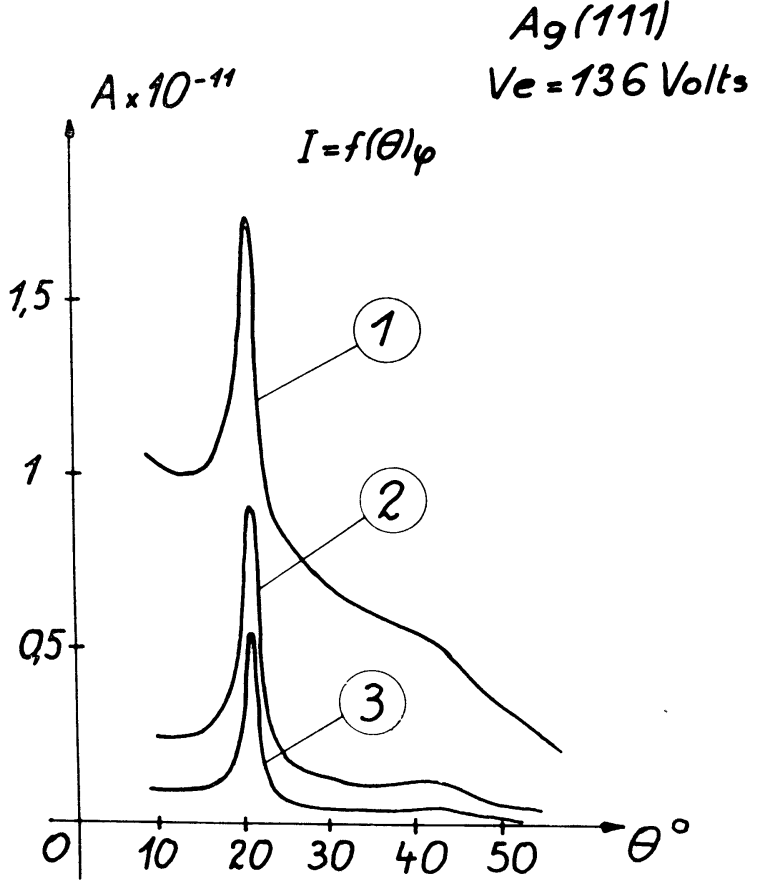

Fig. 8. - Distribution de l'intensité des é'ectrons le long d'un cercle méridien passant par une réflexion de Bragg pour différents potentiels retardateurs. $1 . V_{\mathrm{r}}=0 ; 2 . V_{\mathrm{r}}=101 \mathrm{~V} ; 3 . V_{\mathrm{r}}=134 \mathrm{~V}$.

2. Mesure Des ÉNERGIES. - a) Courbes cumulatives. - Lorsqu'on applique un potentiel retardateur au cylindre de Faraday (Fig. 3), celui-ci fonctionne comme un filtre passe-haut. On peut donc enregistrer des courbes intégrales de la distribution de l'énergie avec le dispositif amplificateur précédant en envoyant l'intensité sur la voie $\mathrm{Y}$ et le potentiel retardateur sur la voie $\mathrm{X}$ d'un enregistreur $\mathrm{XY}$.

b) Courbes de distribution de l'énergie. - $\mathrm{La}$ méthode précédente est malheureusement très sensible aux instabilités du faisceau d'électrons incidents et de ce fait les pertes d'énergie caractéristiques sont souvent noyées dans le bruit de fond.
C'est pourquoi nous avons préféré utiliser pour l'analyse énergétique le système de dérivation électronique couramment employé. On superpose au potentiel retardateur un signal sinusoïdal de faible amplitude et on amplifie uniquement la partie alternative du signal à l'aide d'un amplificateur à détection synchrone ce qui donne la courbe de distribution des électrons en fonction de l'énergie (Fig. 9). Le montage électrique est schématisé sur la figure 10 .

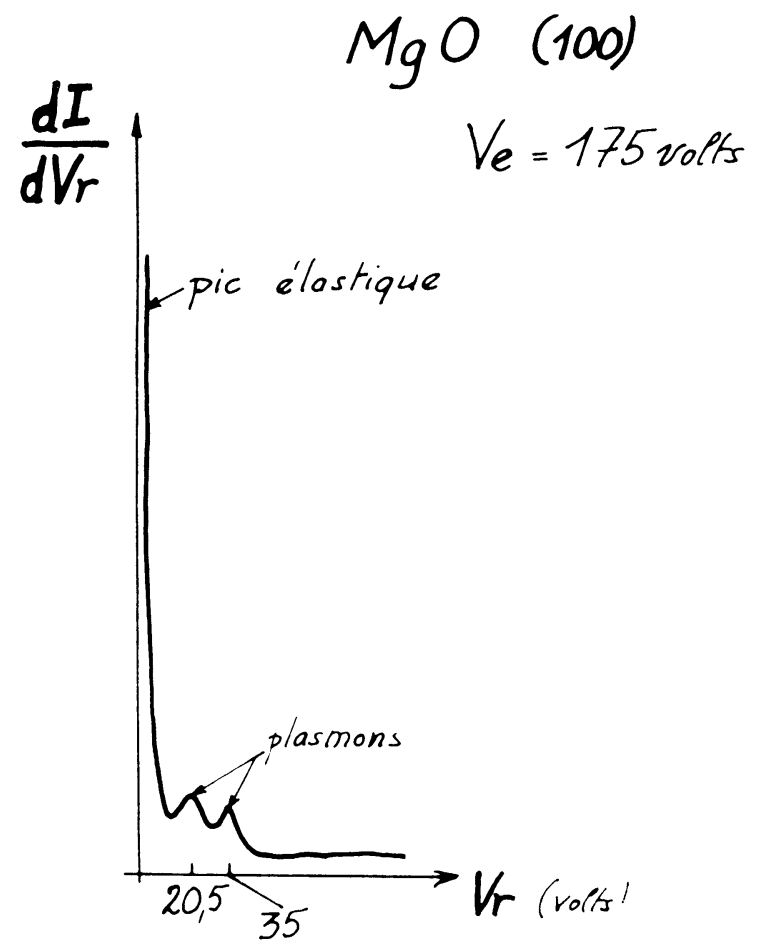

Fig. 9. - Distribution de l'énergie dans une réflexion de Bragg.

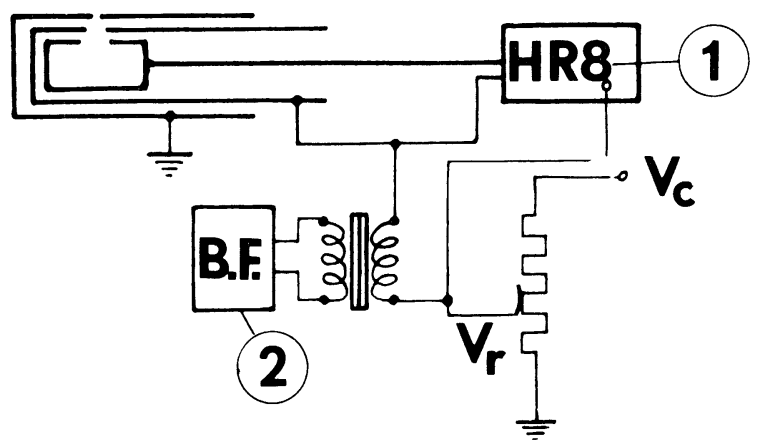

Fig. 10. - Montage différentiateur. 1. Amplificateur de détection synchrone PAR HR $8 ; 2$. Générateur basse fréquence.

Le bruit de fond de l'amplificateur PAR HR 8 utilisé, limite dans les conditions actuelles (fréquence 30 cycles), la sensibilité de l'appareil à quelques $10^{-13} \AA$, ce qui est suffisant pour étudier les pertes d'énergie caractéristiques.

3. Mesures angulaires. - Le repérage de la cage de Faraday est fait d'une part par un tambour gradué fixé sur le conducteur coaxial d'axe $\mathrm{XX}^{\prime}$ (précision de 
lecture $2 / 10^{\mathrm{e}}$ de degré), d'autre part, par une couronne graduée solidaire de l'optique pour le repérage autour de l'axe $Y^{\prime}$ (précision de lecture $1 / 10^{\mathrm{e}}$ de degré). On exclut ainsi les erreurs dues au jeu du système de commande. La lecture se fait à travers la fenêtre d'observation.

III. Premiers résultats obtenus. - Comme première application de cet appareil nous avons cherché à préciser la nature des électrons participant à une réflexion de Bragg et à celle du fond continu au voisinage de cette réflexion. Nous avons pu montrer que comme dans les résultats relatifs aux électrons rapides, il y a dans les réflexions de Bragg en plus d'une quantité prépondérante d'électrons élastiques ou quasi-élastiques une proportion importante d'électrons inélastiques plus particulièrement d'électrons ayant subi des pertes caractéristiques. Dans le fond continu on trouve en plus des électrons inélastiques une certaine quantité d'électrons quasi-élastiques.

La courbe (1) de la figure 8 montre la variation de l'intensité globale des électrons non filtrés se réfléchissant sur la face (111) de l'Ag en fonction de l'angle $\theta$ de Bragg. On voit un pic large $(\Delta \theta=0,07 \mathrm{rd})$ se détachant sur un fond continu important décroissant rapidement avec $\theta$.

Pour mettre en évidence les électrons inélastiques dus aux plasmons qui dans le cas de l'argent présentent des maxima caractéristiques à $3,7,16$ et $25 \mathrm{eV}$, nous avons appliqué un potentiel retardateur de $101 \mathrm{~V}$ ce qui élimine les électrons ayant perdu plus de $35 \mathrm{~V}$.
La courbe (2) de la figure 8 a été obtenue dans ces conditions. On constate que le pic est resté à peu près le même tandis que le fond continu a diminué considérablement. Les plasmons étant diffusés principalement dans la direction du faisceau excitateur qui ici est le faisceau de Bragg, le pic enregistré ne se trouve donc pas modifié (il est toujours composé du faisceau de Bragg élastique + plasmons). En réglant le potentiel retardateur de façon à ne laisser subsister dans le faisceau que les électrons élastiques et quasi-élastiques (potentiel de $134 \mathrm{~V}$ ) on obtient la courbe (3) qui présente un pic correspondant uniquement aux électrons quasi-élastiques, les plasmons étant éliminés. Sa largeur est plus faible $(\Delta \theta=0,04 \mathrm{rd})$.

Il subsiste un faible fond continu dû aux phonons et sans doute aux défauts du réseau cristallin.

L'appareil qui a servi à faire cette étude permet de comparer l'intensité du faisceau incident avec les intensités diffusées et d'en déterminer les valeurs absolues.

Remerciements. - Nous remercions M. Allgayer dont l'habileté et le travail soigneux ont permis de réaliser complètement cet appareil dans notre laboratoire.

Conclusion. - L'appareil décrit dans cet article permet de mesurer électrométriquement la distribution des électrons de faible énergie diffusés élastiquement et inélastiquement dans les différentes régions d'un diagramme avec une bonne précision angulaire. On peut d'autre part, comparer l'intensité des faisceaux diffractés à celle du faisceau incident.

\section{Bibliographie}

[1] Davisson (C. J.) and Germer (L. H.), Phys. Rev., $1927,30,705$.

[2] Farnsworth (H. E.), Schlier (R. E.), GeOrge (T. H.), Burger (R. M.), J. App. Phys., 1958, 29, 1150.
[3] Jones (E. R.), Mc Kinney (J. T.), Webb (M. B.), Phys. Rev., 1966, 151, 476.

[4] Mosser (A.) et Burggraf (Ch.), Rev. Phys. Appl., 1970, 5, 625. 